\title{
EL MOTORISTA: ESTUDIO DE CASO URBANO SOBRE LA CONSTRUCCION DE BARRIOS EN BOGOTA
}

Soledad Niño Murcia*

* Instituto Colombiano de Antropología, Bogotá.

Revista Colombiana de Antropologia, vol XXXII. 1995 
Much of the city of Bogota has been built in an informal manner, the demand for housing having exceed the provisions made by the planning sector and the State. The huge aumbers of people requiring concrete solutions to their problems are no content to wait passively for something to happen; they organize themseives and actively generate a variety of quick and practical "solutions" of their own. Creativity and ingenuity thrive in the face of adversity; links are forged amongst those sharing similar socioeconomic situations; networks are established and relationships strengthened by the pursuit of common objectives. While all family members play a part in this complex process of city formation, the role of the woman has been overiooked and needs to be recognized.

Aunque la antropologia no tiene limites para el reconocimiento de la actividad humana en el desarrollo de aclaptaciones a diferentes medios, la investigación acerca del habitante urbano en Colombia es relativamente reciente, en relación con los estudios especialmente sobre grupos étnicos indigenas.

El ensayo que presentamos, hace parte de la apertura que el Instituto Colombiano de Antropologia inició en 1992 para el examen y conocimiento del hábitat popular urbano.

Los estudios al respecto tienen como objetivo, conocer las diferentes respuestas socioculturales dadas por pobladores urbanos, a la satisfacción de la protección física necesaria a la vida humana, manifiesta en la apropiación de un espacio y en la generación de vivienda. Aunque varie segun diferencias locales y climáticas, la vivienda constituye una de las formas más universales de la cultura material, ya que se encuentra en todas las sociedades no nómadas y representa uno de los componentes de mayor magnitud en el esfuerzo total de construcción de una nación.

Existe una intima relación entre la vivienda y la organización social. Sociológicamente la vivienda tiene un importante papel, pues contribuyea la 
continuidad de la vida comunitaria. Corresponde a la organización familiar construir y desarrollar el hogar, el hogar como unidad de producción y consumo o éstos separados del lugar de socialización y enseñanza-aprendizaje.

La construcción de las casas por especialistas, sólo se da en el Siglo XIX, pues en la mayoría de los casos los miembros de cada familia construían sus propias viviendas. Hoy dia se identifica o los constructores de sus propias viviendas como pertenecientes a sectores narginales.

En todos los países del mundo, la vivienda es un "bien limitado" en términos de Foster (1965) y un alto porcentaje de la población mundial habita en casas que no reunen las condiciones adecuadas, en suburbios de la construcción, producto de acciones de hecho. La vivienda inadecuada trae efectos secundarios por ejemplo en relación con la salud y el bienestar.

En este artículo se resaltarán las diversas formas como los pobladores urbanos satisfacen sus necesidades de vivienda, así como la participación funcional de cada uno de sus miembros. Estus se tratarán como expresión histórica, social y cultural, que además responde a la cristalización de una lógica que rompe y desconcierta, frente a las establecidas por los organismos internacionales y nacionales que se relacionan con el problema.

Examinar un caso, en el marco de con diciones macrosociales y macroeconómicas especificas, permitirá reconocer as adaptaciones y "salidas" propias de un puñado de seres humanos para re lizar sus aspiraciones. Las formas adoptadas, en calidad de estrategias, será ı analizadas.

\section{LA POBREZA Y LAS ALTERNATIVAS AL DESARROLLO}

Al reconocer que existe una amplia misa de población marginada del desarrollo, el Banco Mundial, por ejemplo, sesde fines del decenio de 1980, en sus operaciones de apoyo para el ajuste de la politica social, incluye medidas de producción y fondos sociales para grupos vulnerables. El cambio cuantitativo entre la década del ochenta y la del noventa es significativo: se pasa del $5 \%$ al $25 \%$ de financiamiento total para programas dirigidos específicamente a reducir la pobreza.

Aunque la pobreza se considera como un problema coyuntural, varias estrategias de dicha política han dado por resultado el aumento de la pobreza (Cepal 1993). Durante la década inestable y turbulenta de los ochenta, en me- 
dio de la crisis y los procesos de ajus e a las politicas de desarrollo, el total de pobres en América Latina aumentó ( 160 millones y crecieron en 53 millones los nuevos pobladores en el medio urbano.

Estratégicamente, se hizo invers ón en infraestructura, urbanización y servicios públicos, como "paquete soci l" para incidir en el $46 \%$ de personas en situación de pobreza extrema(Cepa 1993). Es posible concluir hoy, sobre la base del deterioro social, que las or. ntaciones asistenciales y tangenciales de estas politicas, dieron como resultado, la pérdida de control de las condiciones estructurales de la pobreza y la resistencia de los grupos indigentes frente a las lógicas pensadas externamente e impuestas a sus vidas.

Resulta incomprensible, mirado desde los organismos e instituciones cuyos miembros representan las persoilas que han participado de los bienes del desarrollo, reconocer la existencia de estos pobladores del campo o la ciudad que con tan poco, logran vivir y dar respuestas a sus necesidades. Entonces, cómo hacen para seguir existiendo lis que logran existir?

La reflexión que hacemos, no pretende convertir a estas comunidades urbanas tanto en su condición de sujeto como de grupos sociales, en el paradigma de una transformación estructural de la sociedad, ni en los redentores de la historia contemporánea(Max Neef 19.36). Creemos sí, que es necesario enfatizar la "infrahistoria de la vida cotidiana donde las prácticas productivas se entroncan con estrategias colectivas te supervivencia, identidades culturales y memoria popular"(ibid: 64).

Somos conscientes de las limitaciones que encierran factores de demostración que son intangibles, como lo ; recursos no convencionales ampliamente utilizados: la solidaridad, la partcipación, el consenso y el acuerdo. Son recursos que producen relaciones reales, prácticas sociales, organizaciones y también rasgos culturales fundamenales.

La crisis por la que atraviesa Bogotá como capital de Colombia se manifiesta de muchas maneras. Una de las inás significativas es la expansión sostenida de los sectores populares en os últimos años. El desempleo, el trabajo en ocupaciones no asalariadas, la falta de acceso a la educación, a la vivienda, a un terreno, la desnutrición, la violencia intrafamiliar, entre otros, son expresiones de una cara de la moneda. La generación de gran diversidad de estrategias para la supervivencia, es la otr: cara. 


\section{EL MOTORISTA: ESTUDIO DE CASO}

El barrio El Motorista, ubicado sobre a autopista sur de Bogotá, contiguo al cementerio El Apogeo, hace parte del $60 \%$ de la ciudad que de una manera "informal" han desarrollado los llamados sectores populares. Los habitantes del barrio se desempeñaban como motor stas de una empresa de transporte público. El no pago de salarios ni prestaciones a sus trabajadores y el dejarlos sin empleo, motivó la invasión y toma de osesión de un terreno de propiedad de la empresa.

La convicción de su derecho a pagarse la deuda, por las vías de hecho, fue asumida solidariamente también por las esposas, sus mujeres. Ellas comenzaron protegiendo y vigilando organizadamente el lugar mientras los hombres hacían el "rebusque". Como habitantes de ciudad, estas personas tenían en común haber vivido en inquilinatos' o "arimados" a otros familiares. La búsqueda de un espacio y vivienda propia se constituia en un anhelo. Las dos circunstancias anteriores son los ingredientes particulares que se utilizaron cuando apareció "la oportunidad".

En el proceso de apropiación del espa io urbano por vías de hecho (invasión y posesión), se presentan tres mome: tos en los cuales las mujeres participan en forma activa y de diferente manera : ocupación, consolidación y legalización (Meertens 1987: 38).

\section{La invasión: primer momento de ocupación}

El reconocimiento y saber por parte le hombres y mujeres de cómo las mujeres, son miradas y tratadas por la fuerza pública y por las autoridades, les dió la idea para actuar de manera espec fica. Disfrazadas de embarazadas, exaltan su condición de madres y se colocan en las trincheras más peligrosas, como estrategia para avanzar. Las "débile mujeres" con sus "débiles niños", se pusieron como carne de cañón en las confrontaciones, para mantener la

1 Inquilinato es el nombre que se le da a una vivienda compartida por familias cuya organización espacial y social se rige por priıcipios y reglas de convivencia, en la cual una habitación señala un espacio privado, pero la cocina, el baño y un patio son compartidos. Los inquilinatos representan una formade organización para la vida en común. Generalmente en éstos circulan bienes y hay intercambio de servicios. Los desajustes se dan por la extralimitación de pers nas, quienes toman más de lo que comparten, o son intolerantes ante condiciones particulares como niños, enfermos, animales domésticos o a situaciones como borracheras, peleas, etc. 
ocupación del lugar. Hablar, llora, hacer sonar sus ollas y cacerolas, hacen parte del acuerdo endógeno de pla ifícación y de la presentación como cuerpo frente al otro:

...yo me ponía cacerolas en la arriga para parecer embarazada y así lo más probable era que no me pegara los policías, a los señores los echamos atrás y nosotras por delante, nosotras $\mathrm{d}$ fendiamos a nuestros maridos y para pegarles a ellos tendrian que pegarnos $\mathrm{p}$ mero a nosotros y no nos dejábamos. Nos daban a todos un pito y si había problemas, todos a pitar, las mujeres éramos todas muy arriesgadas.

...Mi marido estaba dudoso, no sabia si venirse para el lote o no, y yo ante la posibilidad de tener vivienda pr upia le dije: vámonos y tranquilo que yo peleo, yo participo y hago los turnos de vigilancia que nos toquen. Hasta hice el turno de otros y me pegaban por eso y asi se aseguraba lo del desayuno, yo me las arreglaba a punta de trasnochai

El haber participado en el proceso de obtención del terreno y consolidación de la vivienda y del barrio, constituyó la principal preocupación de sus pobladores y en especial de las nujeres, quienes permanecian todo el día en este lugar. Preocupación no solamente presente durante la vigilia sino también durante los momentos de descanso y de sueño. Esto lo demuestra el relato de una pobladora:

...Una noche hicimos una reuni n porque al otro dia debíamos ir a la Alcaldía y eso si yo me ofrecí para ir porque alguien dijo que en la Alcaldía salió la orden de desalojo. Esa noche yo lloraba y rezaba, casi no pude dormir, pero al rato me cogió el sueño y soñé que se ab ó el cielo y salió la carita de Nuestro Señor, con el cabellito mono y me dijo: yo no sufran más que ustedes se van a quedar con esto, me dijo el propio Nuestr, Señor. Dijo: eso sí van a bregar, pero ya no sufran más, entonces yo me de perté y me agarré a gritar y les dije que Nuestro Señor dijo que esto ya era de riosotros. Al otro día les di la razón a todos y no me creían, pero llegamos a la / icaldia y no encontraron orden de desalojo, entonces si salió lo del sueño. Yo o ví, lo vi lindo, con esos cabellos dorados y los ojos azules...

Los trabajos que requiere lo mar una vivienda, bien justifican la cantidad de esfuerzos que debieron realizur en su jornada laboral; los hombres en lugar de llegar a sus casas a descansar, llegaban a asumir otra serie de trabajos: participar en reuniones nocturns para solucionar problemas comunitarios, asistir a cursos de capacitación y planear nuevos trabajos a desarrollar, o ejecutar oficios domésticos. Implicaba grandes esfuerzos que se realizaban con la motivación de lograr la conso idación del barrio y su legalización. 
Nosotros establecimos turnos para cuidar ce noche, quitábamos los cojines de los asientos de los buses y los metiamos a a carpa para que durmieran los que no tenían que cuidar. Haciamos turnos de 2 horas y al terminar despertábamos a los del nuevo turno y nosotros a dormir en los cojines.

En esta etapa los "invasores" como tradicionalmente se les llama invierten más o menos, cinco años de su tiempo. Bajo el refrán’la constancia vence” este grupo humano sabe por tradición familiar que ésa es la única vía para lograr un lote, una vivienda y un barrio.

El proceso de consolidación del proyec implicó la realización de acciones todas orientadas a lograr el objetivo. Se t itaba de acciones de apoyo mutuo que tienen que ver con la transmisión y sostunimiento del valor y la fortaleza, necesarios para sacar adelante la empresa y para superar momentos de temor y miedo. La combinaciónde tareas individuaies y colectivas va creando y realizando el proyecto comunitario, en la med da que pudieran sortearse una y otra vez las situaciones de confrontación.

La existencia de una división del trabajo yor género, tradicionalınente asumida, se modifica en el barrio cuando las nujeres realizantareas clasificadas como masculinas. Esta división no sólo esté expresando el aspecto de fuerza física, sino una nueva manera de compartir s $n$ diferencias el esfuerzo necesario a la concreción del fin, que en estecaso ticne que ver con autoridad y capacidad de luchar públicamente:

... aqui la mujer ha luchado hombro a hombro con el hombre. Nosotras cargamos plaquetas, materiales, ayudamos a hacer la rancha [rancho] y a todo lo que se presente... fuimos las que hicimos los hueces para el alcantarillado. Ibamos por los postes, recuerdo un 31 de diciembre que poniendo un poste de la luz a las 11 de la noche y cuando ya casi lo teníamos coronado, el poste se cayó y casí me cae en las espaldas, Virgen purísima, me hubiera matado.

\section{La Consolidación}

El reconocimiento social de que de allí y: nadie los sacaría, creó condiciones parair transformando el lugar comunitari mente y sobre la base de acuerdos explícitos consolidar la estructura indivi lual y grupal para un barrio.

Estos acuerdos fueron: condiciones igual spara resolver todos el acceso a la vivienda; condiciones desiguales para fav ilias desiguales en términos del 
promedio de sus miembros; localización espacial a la suerte (lo importante era que todos reconocieran que tenian iq ualdad de oportunidades); reconocimiento de la autoridad de una junta que reparaba propuestas y las justificaba.

Los pobladores tenían claro que "tener casa no es riqueza, pero no tenerla es tremenda pobreza".

\section{La Legalización}

La legalización se constituye en parte de una nueva lucha, que representa a la vez esfuerzos para adquirir la e critura de su propiedad como documento público. La cual simbólicamente, si nifica el fin de esta lucha. La legalización de la propiedad es tarea de los homt es. Ellos buscaron que la escritura de "su propiedad" figurara a su nombre. C ntrario a lo pensado por versiones feministas, ésto no molestó a las mujerc, quienes piensan que los hombres tienen más habilidades y paciencia para "perder el tiempo haciendo colas". La junta administradora propició un cambio para esta visión y actitud al buscar que la pareja compartiera el derecho de propiedad y exigir que las escrituras aparecieran a nombre de ambos cónyuge :

\section{La vivienda como medio par., la construcción de comunidad}

El proceso llevado a cabo en es e terreno, para hacer un barrio conformado por viviendas y espacios público; para sus habitantes, transpasó el fin en si mismo, convirtiéndose en un gran medio de gestación de comunidad. Esto quiere decir, agruparse para el logr de propósitos comunes. El trabajo en las diferentes etapas permitió conocers ' más, crecer en solidaridad y gozar de los beneficios de la comunidad: "todos para uno; uno para todos". El trabajo también permitió realizar una serie de cambios, con respecto a la concepción del rol femenino y masculinoy tambiè al de la familia. La subestimación de la mujer como constructora y su tesó para resistir la fuerza pública, se vieron modificados en el "laberinto"? de los hombres.

Aqui en el barrio, en El Motorista nosotros vimos cómo las mujeres se fueron integrando y veo que antes las señoras estajan muy intimidadas en las casas, pero aqui han tenido como más li' eración, pues antes, uno llegaba y las cogia a patadas, pero ahora ellas no se de an

2 El laberinto segun Wallace (1901) se refiere al conjunto de elementos que unifican las representaciones del individuo 
También en el "laberinto" dc las muje ss se dió una modificación.

...yo era muy timida, no conocía casi a ri idie y no tenía amistades, pero aquí aprendi a hablar y a meterme en todas par es. Donde había reunión ahí estaba y donde habia que pelear peleaba y si habi que gritar cuando lo de la negociación, pues eso hacía...

\section{La utilización de recursos no conv ncionales para construir comunidad.}

La utilización de recursos no convencic nales(Max Neef 1986) por parte de estas personas, más que resolver una nece idad por medio de la unión de esfuerzos para la construcción del barrio, perinitió encontrar, que a diferencia de los recursos convencionales como el capital que si se gastan, habian crecido como seres humanos en la medida en que tenian más conciencia social, más solidaridad, más apoyo y fortaleza.

El Motorista presenta el rostro de un berrio organizado;cuenta conlos servicios completos, está legalmente incorpor:ıdo al tejido urbano, ha construido comunidad, en la cual existen organizaciones comunitarias como: la Asociación de Mujeres, la cooperativa, la Junta i : Acción Comunal, la junta de padres y variosgrupos deportivos.

En la consolidación del barrio se unel agentes externos de instituciones privadas y estatales que ofrecen capacitac in, lo cual representa más cultura organizativa y capacidad de gestión, más c eatividad popular y capacidad de ayudarse mutuamente y más calificación y sonocimientos a partir de la experiencia. Estos recursos no convencionales queaumentan cuando más se utilizan, se constituyen en la base para iniciar el proceso de construcción de las viviendas.

En la construcción de viviendas la gent $>$ del barrio reunida como comunidad reconoce los requisitos particulares pa a que los individuos como cabeza de familia puedanacceder a créditos del si tema financiero. Para ello se requiere tener un trabajo permanente o conta: con un fiador con propiedad raiz, también haber dejado recursos económicos en una cuenta bancaria de ahorros durante un tiempo y tener buenas referencias. Recordemos que la mayoría de los habitantes del lugar tienen trabajos "informales", relacionados con la conducción de vehículos principalmente. 
Quienes no logran el acceso a ios créditos establecidos por el sector bancario, se ven en la necesidad de cear formi s alternativas que den respuestas inmediatas. Es evidente el gran in genio matifestado en la diversidad de respuestas ante la adversidad, basada : en la solidaridad y surgidas alcompartiruna situación socioeconómica similar.

En El Motorista ante la inestal ilidad laboral y la necesidad de mejorar los ingresos familiares, la mujer aprovecha los lazos de amistad entre vecinos para organizar "cadenas". Las cadenas son una forma común de cooperación económica, que sirve al mismo tiempo para reforzar lazos de unión entre sus miembros y contribuye a fortalecer el gy apo. Permite cohesión de los integrantes de la red, a partir de relaciones bas adas en la confianza y la reciprocidad. Es similar al sistema de "tandas" de scrito por Larisa Lomnitz (1983: 94) para Méjico y por Clifford Geertz (19 2) para algunas regiones de Asia y Africa. Lomnitz relaciona la economíade los marginados de la ciudad con las bandas de cazadores y recolectores, en el sentido que gracias a la destreza, astucia y solidaridad social pueden afrontar problemas de superviviencia, de ahorro en grupo y suplir necesidades de carácter inmediato.

Las cadenas son una forma de cooperación económica, practicada frecuentemente por los habitantes de los barrios populares;se basa en una ideología de ayuda mutua y consiste en que veinte o veinticinco personas se asocian para aportar cuotas quincenales de $\$ 10.000$ a $\$ 50.000$ para conformar un fondo común que se rotará entre sus integrantes.

Así pues, si veinte personas empiezan la cadena en enero, con una cuota quincenal de $\$ 50.000$, se asignará el millón de pesos recogido cada vez, a cada uno de sus integrantes, por turnos, de manera que el ciclotermina en el mes de octubre.

Existen tres formas de distribución de los recursos. La primera establece mediante un sorteo quién será el pióximo en recibir la cadena y este nombre ya no concursará en el siguiente; se mantiene la expectativa cada quincena. La segunda consiste en que la perscina organizadora de la cadena establece con anterioridadlos "puestos fijos", r servando para sí el primer puesto y para los demás un orden según se vayan aıotando. ia tercera posibilidad es hacer una reunión inicial con todo el grupo, y por sorteo se reparten los turnos de toda la cadena con las fechas correspon ientes. Si a alguien le correspondió uno de los últimos turnos y necesita el cinero antes, lo puede negociar con los participantes del grupo a quienes les correspondieron las fechas cercanas; se cambian favores que se retribuyen en un futuro. A veces se hacen rifas de electro- 
domésticos, como un estímulo para los que ran quedado en los últimos puestos.

Esta forma de ahorro no implica intereses, no requiere tesorero, ni documentos escritos, sólo se basa en relaciones c e confianza y amistad entre vecinos, amigos o parientes.

Tampoco se presentan casos de incumf iimiento a lo pactado, pues si en algún momento alguien no puede apo tar su vuota, otra persona la dará con el compromiso que en la siguiente fecha de pa o le correspondendos a quien no pudo pagar la vez anterior. Generalmente, , uien ingresa a una cadena no se retira una vez concluido el ciclo, sino que cinicia una nueva o participa en varias simultáneamente. En la mayoria de lo: casos el monto obtenido por las cadenas se emplea en el mejoramiento de 1 ; ; viviendas. Es de anotar aqui la característica de la consolidación de las con rucciones, por etapas, de manera progresiva, de tal forma que desde la instal ción de "la rancha" se está pensando permanentemente en hacerle reformas y ampliarla a medida que crece la familia.

\section{Autocstima, autovaloración y perte encia}

El reconocimiento del logro, de la capacidad y tenacidad por haber logrado un barrio, es fuente permanente de autoe tima colectiva y autorrealización individual por género y grupo.

La organización social en este barrio, in cluye la categoria "los propios" y"los nuevos" para diferenciar a los primeros pobladores, de quienes han llegado en otras condiciones. Para los "propio." o fundadores la relación establecida con el territorio y el espacio apropi do, asi como con la vivienda es muy significativa: "no habia nada y logramo todo". Este logro, plasmado en la consecución de una vivienda es resultado c cl trabajo de varios años y entraña luchas e historias plenas de significado. P. ra sus pobladores no es una simple construcción y le atribuyen afectuosamen:e un carácter femenino, denominándola "rancha".

Por el carácter de su ocupación como c iductores de transporte urbano, deben cubrir distintas rutas y recorrer exten os sectores de la capital, por lo cual tienen un amplio conocimiento de la ci lad y reconocen en ella caracteristicas particulares que les permiten identif ar claramente diferentes territorios. Es asi como califican sectores del ban " utilizando categorias de otros 
sectores de la ciudad. Es como si el barrio condensara la totalidad de la ciudad, es ver lo macro en lo micro.

Asi pues, la calle en donde e localizan las casas de los miembros de lajunta la denominan "El Chicó" (nombre de barrio bogotano que tradicionalmente se conoce como en el (we habitan personas de la "clase alta"); la calle más amplia en donde pueden circularvehiculos se denomina "Chapinero" (se asocia con el mimiento vehicular y la presencia de gran cantidad de personas que perma entemente transitan por el sector). Generalmente en cada cuadra hay una ienda pero la que tiene cuatro se denomina "El Restrepo" (por hacer ahısión a la concentración del comercio del sector) e igualmente denominan El Cartucho" a la calle más estrecha, más parecida a un callejón (se relacic na con pobreza y deterioro) en ésta, comparativamente con las otras viviendas del barrio hay algunas que todavía están en estado de "rancha".

De tal forma la ciudad tiene centido y significado para sus habitantes, no es sólo espacio físico, es espacic transformado en territorio por sus mismos pobladores. El apego y sentido de pertenencia se expresan en el siguiente testimonio:

Si a mi me saliera una casa en t tra parte, pues claro que la compraria pero me no me iria para allá, este barrio o hemos hecho nosotros, esto es un pedazo de la vida de uno.

Hemos trabajado sólo algunc; aspectos de las formas como los sectores populares suplen necesidades pa: a obtener satisfacciones como seres humanos. La capacidad de solidarizars, de luchar, de organizarse y forjar la voluntad para obtener un bien, son sól algunos de los recursos con que cuentan y que los caracterizan particularme ite.

El Motorista refleja hoy en sus viviendas y en la organización de barrio, la cultura popular de un se ior de Santafé de Bogotá. Indudablemente la participación de la mujer en ci proceso de consecución de la vivienda ha sido muy significativa, no sol: nente en el barrio El Motorista, sino en muchos otros sectores de la capilal y de otras ciudades del pais. Es la mujer quien toma la iniciativa, al ser , lla quien afronta las dificultades domésticas y permanece la mayor parte del tiempo, en el espacio que habita con sus hijos. Es por esto que compromete de manera firme a su compañero para lograr este objetivo. 
Por las dificultades que representa su papel en esta lucha, adquiere un importante significado, al involucrar a todos y a cada uno de los miembros del grupo familiar. La labor femenina no ha side reconocida por el Estado, a través de las políticas referentes a mujer y vivie da, por lo que es justo tener en cuenta su verdadero papel, tanto al interior le la unidad doméstica como dentro del proceso de hacer ciudad.

\section{BIBLIOGRAFIA}

CEPAL

1993 Pobreza, ajuste y equidad. Consejería presidencial para la política social. Bogotá.

FOSTER, George

1971 Peasant society and the image of the limited good. En Man in adaptation: the institutional frimework: 298-310, Ed. Yehudi A. Cohen. Chicago, Aldine.

GEERTZ, Clifford.

1962 The Rotating Credit Association: a 'middle rung' in development, Economic development and : cial change, Vol. 10, N³: 241263.

LOMNITZ, Larissa.

1983 Cómo sobreviven los marginados. Editorial Siglo XXI, México.

MAX-NEEF, Manfred

1986 Desarrollo a escala humana. Una opción para el futuro. Cepaur, Hammars Kjold, Grafisca AB Motala, Chile.

MEERTENS, Donny.

1987 Mujer y vivienda en un barrio de invasión. Revista Foro, No. 4, Noviembre: $38-46$. Bogotá.

WALLACE, A. F. C.

1961 Culture and personality. Ranciom House. New York. 